\title{
L'invraisemblance du surnaturel
}

Fiction et réalité dans un culte bouddhique birman

\section{Guillaume Rozenberg}

\section{(2) OpenEdition}

Journals

Édition électronique

URL : http://journals.openedition.org/assr/21066

DOI : $10.4000 /$ assr.21066

ISSN : $1777-5825$

Éditeur

Éditions de l'EHESS

Édition imprimée

Date de publication : 1 janvier 2009

Pagination : 129-146

ISBN : 978-2-7132-2215-3

ISSN : 0335-5985

Référence électronique

Guillaume Rozenberg, «L'invraisemblance du surnaturel », Archives de sciences sociales des religions [En ligne], 145 I janvier-mars 2009, mis en ligne le 01 janvier 2012, consulté le 20 avril 2019. URL: http://journals.openedition.org/assr/21066; DOI : 10.4000/assr.21066 


\section{Guillaume Rozenberg}

\section{L'invraisemblance du surnaturel Fiction et réalité dans un culte bouddhique birman*}

Supposez un ethnologue travaillant sur le fait religieux dans une contrée lointaine. Il est confronté, sur le terrain, à un phénomène insolite et incroyable ; une affaire d'hommes volants, par exemple. La chose est courante dans le métier, presque un classique du genre. Il existe des solutions éprouvées qui permettent de rendre compte du plus extraordinaire. Aussi s'agit-il d'une situation où il n'y a, au fond, pas de problème. L'ethnologue, armé du sens commun propre à la profession, adopte spontanément une posture adéquate. Mais arrive un moment où l'insuffisance de cette posture lui devient évidente : une partie de la réalité s'en trouve voilée, des faits sont gommés qui paraissent pourtant décisifs. $\mathrm{Ne}$ lui faut-il pas repenser sa manière de voir ? Et tant pis si l'approche et l'interprétation qu'il formule sont insatisfaisantes. Au moins, la difficulté aura été signalée, le regard que porte l'anthropologie sur les expériences du surnaturel aura été interrogé.

Qu'on se rassure : mon intention n'est pas d'affirmer l'existence du surnaturel - l'ethnologue reste ethnologue. Voici, pour être précis, les données du problème. Depuis plusieurs années, je mène des recherches sur un culte bouddhique birman, un culte de prospérité et de salut dédié à des personnages aux facultés extraordinaires. Ces personnages, sortes de "superhommes ", manifestent leur puissance surnaturelle en accomplissant divers prodiges. Ils visent par ce moyen à convaincre les fidèles qu'ils sont bien ce qu'ils prétendent être. Or leur mise en scène ne s'avère pas au point, tant s'en faut. Des anomalies criantes ponctuent leur représentation, anomalies qui soulignent la mystification. Nonobstant, nombre de Birmans croient dur comme fer en ces personnages. Curieuses gens, qui prennent pour vrai et authentique ce qui est visiblement faux et fabriqué. L'anthropologie, cet assortiment de schèmes de compréhension destinés à faire sens de l'étrangeté culturelle,

\footnotetext{
* Cet article n'aurait pas vu le jour sans les encouragements de Marlène Albert-Llorca. Je sais gré, d'autre part, à l'ensemble des participants au groupe de travail sur les expériences du surnaturel pour leurs remarques sur une première version du texte (notamment Sébastien Tank, invité à discuter les différentes contributions, et Grégoire Schlemmer, commentateur exigeant de mes écrits). Sophie Albert a assuré l'édition critique du texte final.
} 
offre plusieurs façons de résorber le paradoxe. Toutefois, ces solutions m'apparaissent impropres, et je soutiens qu'on est bien face à un paradoxe, qui dit quelque chose sur le rapport au surnaturel. Au lieu de réitérer l'interrogation anthropologique standard - « comment peut-on croire à l'incroyable ? " -, il convient de se demander : "Comment peut-on croire à l'invraisemblable ? "

L'invraisemblance du surnaturel pose la question de la croyance. Le terme, on le sait, est d'un usage ambigu. L'idée de croyance constitue d'un côté une catégorie descriptive dont les ethnologues se servent pour exposer les conceptions indigènes, dans les domaines de la religion et de la magie notamment : " La croyance, chez les Birmans, veut qu'un moine ayant atteint l'état de sainteté ait le pouvoir de veiller sur les fidèles et de les préserver de l'infortune ». Dire que c'est une croyance revient à dire qu'il s'agit d'une représentation collective ou fait de culture. Le terme de croyance ainsi compris n'admet pas d'antonyme : parler des «incroyances " d'une société n'a pas de sens - les croyances varient d'une société à l'autre et, au sein de chaque société, elles forment un ensemble structuré. Bref, la ou les croyances, dans cette première acception, sont des propositions relatives à la vision du monde qu'une société entretient (propositions susceptibles d'interprétations et de prolongements divers selon les individus ou groupes sociaux) ${ }^{1}$. L'idée de croyance renvoie de l'autre côté à un type de jugement et d'attitude concernant un phénomène ou un être vis-à-vis desquels l'incrédulité, le doute, l'indifférence sont possibles : "Un ami birman m'a raconté comment était née sa croyance dans la sainteté de ce moine ». Tous les membres de la société ne partagent pas ladite croyance ; rien ne les y oblige. La croyance, dans cette seconde acception, est un fait d'opinion individuelle et une prise de position motivée (on croit pour telle ou telle raison). Elle s'articule sur la croyance collective (dans le cas de mon ami, sur la conception birmane de la sainteté), qui en est comme le socle. Mais les deux ne se situent pas sur le même plan, elles se rapportent à des niveaux distincts : le niveau primaire du régime culturel de représentations, coextensif à la collectivité considérée, et à l'intérieur de cette communauté de croyances, le niveau secondaire de ce que, pour éviter toute confusion, je nommerai le "croire ", marqué par la variabilité et l'engagement individuels ${ }^{2}$. La langue birmane, mieux pourvue sur ce point que la nôtre, dispose de deux verbes, avec les substantifs qui en dérivent, pour exprimer la distinction. Les Birmans regardent comme allant de soi l'existence de ces sortes de

1. Pour une tentative d'explicitation du contenu de la notion de "croyance » lorsque celleci est appliquée par les ethnologues aux représentations religieuses d'une société, $c f$. Southwold (1979).

2. Ce choix de vocabulaire est fait, précisons-le, sans souci des manières dont d'autres ont défini et employé le terme de " croire ». Ainsi, exemple parmi les plus connus, Danièle HervieuLéger, s'inspirant des écrits de Michel de Certeau, confère au terme une extension sémantique maximale : le "croire », pour Hervieu-Léger (1993 : 105-106), englobe ce que, pour ma part, je juge nécessaire de distinguer sous les termes de «croyance » d'une part et de «croire » de l'autre. 
superhommes qu'ils appellent weikza, ils tiennent l'état de weikza pour réel ou possible, sans doute aucun : ils croient (yuhsa-) aux weikza. Cependant, certains d'entre eux n'accordent nul crédit aux superhommes objets du culte qui m'intéresse, quand ils ne les donnent pas pour des imposteurs : ils ne croient (yonkyi-) pas à ces weikza. Entre la croyance (ayuhsa) et le croire (yonkyihmu), il n'est pas de relation mécanique; l'une n'engendre pas nécessairement l'autre. Je m'efforce, au travers de mes recherches sur le culte, de cerner le processus qui conduit un individu de la croyance au croire. Je voudrais saisir comment et à quelles conditions s'effectue la conversion, aussi bien que pourquoi elle échoue parfois, l'incrédulité persistant (il est indispensable, pour appréhender le croire, de prendre en compte le point de vue des incrédules). Que le basculement dans le croire implique une expérience du surnaturel, voilà qui a été souvent relevé, décrit, analysé. Que l'expérience en question puisse présenter un caractère invraisemblable, voilà qui n'a peut-être pas reçu l'attention méritée.

L'idée ici défendue d'une indispensable distinction, dans le vocabulaire et l'analyse de l'ethnologue, entre "croyance " et "croire ", ainsi que le projet de fouiller le processus d'évolution de l'une à l'autre, ont pour origine l'étude des représentations et des discours birmans. Néanmoins, il faut l'indiquer, la démarche pourrait se révéler d'une pertinence plus large et s'appliquer à d'autres contextes. À lire les travaux de référence sur la «croyance " en anthropologie (pour m'en tenir à ce que je connais un peu) ${ }^{3}$, on s'aperçoit que leurs auteurs : - soit, tels E. E. Evans-Pritchard (1937) et Jeanne Favret-Saada (1995 [1977]), se sont focalisés sur les modalités de la croyance - et par conséquent du scepticisme - dans un contexte donné sans parvenir à énoncer nettement la distinction entre les deux niveaux de la croyance et du croire, entretenant de ce fait une certaine confusion conceptuelle ;

- soit, tels Rodney Needham (1972) et Jean Pouillon (1993), ont produit une critique, à caractère relativiste, de l'usage fait par les ethnologues de termes aussi polysémiques et incertains que "croyance " (substantif) et "croire " (verbe), plaidant alors - de manière excessive et selon des arguments contestables - pour un renoncement total ou partiel à l'usage de ces termes en anthropologie ;

- soit encore, tel Dan Sperber (1982), se sont attaqués de manière décapante au point de vue de l'anthropologie culturelle ou sociale sur les représentations ou croyances indigènes, pour arriver cependant à une formulation étrangère aux cadres de cette anthropologie, une formulation ignorante des mécanismes socioculturels qui importent à l'ethnographe parce qu'ils s'imposent à son observation ; - soit, enfin, tels Octave Mannoni (1969) et surtout Jean Bazin (1991), se sont livrés, sur un mode tournoyant, à de stimulantes méditations sur le problème de la croyance, méditations toutefois pas entièrement systématisées, avec l'absence, notamment, de mise en place d'une terminologie en adéquation avec leurs constats.

3. Sur ces travaux, voir la synthèse de Lenclud (1990). 
La distinction entre "croyance " et "croire ", et le fait de placer au centre de la réflexion le processus d'évolution de l'une à l'autre, constituent des éléments qui à la fois rejoignent les préoccupations de ces auteurs et autorisent, me semblet-il, à sortir de certaines des impasses dans lesquelles ils entraînent l'ethnologue ou, en tout cas, à dépasser les limites de leurs propositions. Mais c'est là, évidemment, une autre discussion.

\section{D'étranges superhommes}

Tout commence il y a plus d'un demi-siècle, en 1952, dans un village de Birmanie centrale. Cette année-là, un paysan de vingt-six ans, quasiment analphabète, est soudain possédé par des weikza. Les weikza, personnages clés dans le bouddhisme birman, sont des humains qui ont acquis des pouvoirs extraordinaires, dont la faculté de prolonger leur existence au-delà de sa durée normale, jusqu'à l'âge de mille ans pour certains, jusqu'à un âge indéfini pour la plupart. Outre réaliser leur ambition propre, qui est d'accéder au nirvana, ils ont vocation à protéger et propager la religion bouddhique (Rozenberg, 2005 : 87-122). Lorsqu'un individu est parvenu à l'état de weikza, il ne meurt pas. Il " sort » : il se volatilise et part résider dans un lieu invisible réservé aux êtres de sa qualité. Une fois «sorti ", il peut rentrer en relation avec le monde afin de poursuivre son œuvre de salut. La communication s'établit, entre autres, par la voie de la possession. Le weikza élit un individu pour médium ; par ce truchement, il délivre un enseignement sur la voie du nirvana et transmet sa bénédiction aux fidèles en favorisant leur réussite dans tous les domaines - un weikza agit à la fois comme pourvoyeur de bienfaits (richesse, fécondité, etc.) et comme redresseur d'infortune (guérison de maladies, etc.), éléments essentiels au développement de son culte. Ainsi en va-t-il pour notre jeune paysan. Celui-ci, possédé par un groupe de quatre weikza âgés de cent cinquante à mille ans, diffuse leur enseignement et leur pouvoir. Un culte voit le jour. Une particularité remarquable distingue toutefois ce cas des nombreux autres cultes de weikza en activité : les quatre weikza, non contents de posséder leur médium, apparaissent régulièrement en chair et en os.

Aujourd'hui, ces apparitions ont lieu au monastère fondé par le médium (devenu moine dans son village en 1973). Des fidèles se pressent de toute la Birmanie pour voir les weikza et leur rendre hommage. Les séances sont quotidiennes en période d'affluence. Elles débutent vers 19 h 30-20 h, après la tombée de la nuit. Elles se déroulent à l'étage d'un des édifices du monastère. Il n'est pas permis de rester en bas et d'observer les choses de l'extérieur. Cela a son importance, puisque le premier weikza est supposé arriver en volant. Il vient de son merveilleux séjour, la Montagne des Dragonnes. Son extraordinaire entrée en scène est très attendue car elle est la démonstration par excellence de ses facultés supra-normales, elle est la preuve manifeste qu'il est un «vrai weikza» 
(weikza asit). Le personnage surgit par l'embrasure d'une fenêtre dans le coin avant gauche de la salle. Cette fenêtre se trouve à trois ou quatre mètres de hauteur. S'il est censé voler, c'est-à-dire se déplacer debout en lévitation, le weikza ne se meut pas, en réalité, en volant. La discordance suscite d'ailleurs un commentaire sceptique chez certains spectateurs. On distingue d'abord des mains qui s'agrippent de l'extérieur au châssis de la fenêtre. Puis un visage se détache dans l'encadrement et le personnage bondit à l'intérieur. Il entame alors une sorte de prêche à l'adresse de l'assistance. Ses traits rappellent ceux du médium du culte, lequel n'est jamais présent au cours des séances d'apparition (photos 10 et 11). Quand bien même son costume blanc (agrémenté d'un turban) et le peu de visibilité (dû à l'obscurité) laisseraient planer un doute, sa voix et son parler lèveraient toute ambiguïté.

À plusieurs moments pendant le prêche, des noix de coco et des bougies tombent surnaturellement, pour ainsi dire, au milieu du public. Elles sont apportées et jetées de manière invisible par les weikza. Emplies d'énergie bienfaisante, elles sont sources de succès, de bonne santé et de prospérité. Les fidèles s'en emparent avec fièvre. Certains guettent leur chute pour les récupérer avant les autres. Les plus attentifs aperçoivent une trappe qui s'ouvre dans le faux plafond, puis un bras et une main qui lancent une noix de coco ou des bougies - objets dont ils ne se saisissent pas moins avidement. Les noix sont cueillies dans la journée au vu et au su de tous, au sommet des cocotiers du monastère.

Le weikza assortit son discours de prodiges dans le but d'affirmer sa puissance. Une première démonstration atteste derechef sa capacité de voler. Le personnage choisit des fidèles parmi ceux qui n'ont jamais assisté à une séance. Il les invite à examiner la fenêtre par laquelle il a surgi. Les fidèles se penchent à l'extérieur. Ils constatent que la hauteur est telle que nul ne saurait sauter jusqu'en bas sans se casser le cou et que rien ni personne n'est là pour faciliter le trajet aérien du weikza. Ils sont renvoyés à leur place. Le weikza saute ou plutôt vole à travers l'ouverture, en disparaissant de la vue du public. Il réapparaît quelques secondes plus tard par la porte d'une petite pièce située dans le coin avant droit de la salle. La performance sera accomplie une autre fois pendant la séance, la sortie et la réapparition s'effectuant en des points différents.

Un second type de prodige consiste à opérer la translation d'un objet entre deux personnes. Le weikza demande à un fidèle un morceau de tissu, mouchoir ou foulard. Il y enveloppe un petit objet, puis frotte le paquet entre ses mains. Il le remet au fidèle qui va s'établir à un endroit de la salle. Un second fidèle fournit un autre morceau de tissu que le weikza prend dans ses mains pour en faire une boule avant de le lui rendre. Tout est en place et le premier fidèle, sur instruction du weikza, énonce le vœu suivant: "Que l'objet qui est avec moi arrive dans l'autre morceau de tissu ! » À la suite de quoi, vérification est faite : l'objet est bien passé. 
Lorsque le weikza a fini de prêcher, il quitte la salle par la fenêtre, en volant. La séance se poursuit avec l'apparition des trois autres weikza. Ils entrent en marchant, à partir de la petite pièce du coin avant droit ; cette pièce est une sorte de sas entre leur séjour invisible - la Montagne des Dragonnes - et le monde normal. Contrairement au premier weikza, laïc (d'où son habit blanc), ceux-là sont moines (portant une robe ocre) et donc soumis au code de discipline monastique qui interdit d'exhiber ses pouvoirs. Ils se conduisent avec retenue pendant les séances. Ils sermonnent les fidèles et leur transfèrent de l'énergie de réussite en les tapotant ou en récitant des formules.

L'un de ces trois moines weikza et le weikza laïc ne s'adressent jamais ensemble au public, et tous deux, le moine et le laïc, ressemblent à s'y méprendre au médium du culte. Autrement dit, le médium joue le rôle de deux des quatre weikza, les deux plus importants. Néanmoins, on s'attache, via un expédient, à convaincre du contraire. Peu après que le moine weikza est apparu et pendant qu'il prêche au milieu de la salle, le weikza laïc se montre de nouveau : il se tient à l'entrée de la petite pièce, sans s'avancer. Les traits imperceptibles en raison de son turban bien ajusté et de sa position latérale par rapport à l'assistance, il reste là coi une ou deux minutes avant de disparaître dans la pièce.

La séance, qui dure une heure et demie en moyenne, se conclut par le départ des weikza. Ils se retirent par la porte de la petite pièce, se dérobant à la vue du public.

\section{En quête de raison}

Longtemps, j'ai refusé de m'interroger sur le caractère invraisemblable des apparitions des quatre weikza. Il est en effet un principe de neutralité morale qui dicte à l'ethnologue de mettre en suspens tout jugement sur le croire de ses interlocuteurs. S'ils disent croire, il faut croire, sinon comme eux, du moins avec eux, soit reconnaître la légitimité de leur conviction et en rechercher les ressorts sociaux ou culturels. L'ethnologue doit, à partir des discours et des actions indigènes, tâcher de comprendre comment un culte se construit, ce que ce culte exprime et ce qu'il rend possible; il doit raccorder le phénomène au système de représentations dont il procède et le situer au sein du complexe religieux dans lequel il s’inscrit. Aussi écartais-je comme non significatifs les éléments qui indiquaient l'étonnante crédulité des adeptes des quatre weikza, qui dénonçaient l'imposture des personnages en lesquels ils croient - un croire on ne peut plus sérieux puisqu'une cérémonie exceptionnelle, destinée à prolonger au-delà de mille années la durée de vie de chacun des quatre weikza et déjà accomplie pour trois d'entre d'eux, met en danger de mort ses participants, du fait de la déflagration qu'elle implique.

Or, malgré l'activité déployée par les weikza pour faire croire, c'est-à-dire pour administrer la preuve de leurs pouvoirs, malgré l'accent mis par les adeptes 
sur le caractère probant de cette démonstration qu'ils désignent comme le fondement de leur adhésion au culte - une adhésion raisonnée découlant, expliquentils, d'une expérience de première main (lettway), d'une étude des faits (layla-), d'une mise à l'épreuve des weikza (san-) -, bref, malgré un mode de pensée en apparence tout entier articulé sur la rationalité empirique, l'invraisemblance de la représentation demeure patente. L'invraisemblance est patente et elle semble même, dans une certaine mesure, assumée, sinon affichée (la collecte, le jet et la réception des noix de coco). Voilà donc une manifestation du surnaturel ostensiblement invraisemblable et à laquelle pourtant des gens croient, c'est-à-dire qu'ils considèrent, en dépit du bon sens, être en présence de "vrais weikza ", capables, grâce à d'extraordinaires facultés, de leur assurer le salut tant temporel que spirituel, de leur délivrer des bienfaits et de les conduire sur la voie du nirvana.

Une fois le paradoxe isolé, il faut se demander si l'anthropologie ne suggère pas une solution pour le surmonter. Ne se tarauderait-on pas inutilement avec un faux problème ? J'entrevois au moins quatre formules anthropologiques susceptibles de résoudre l'équation et de restaurer un ordre de la raison. Ces formules ne sont pas mutuellement exclusives, elles peuvent se recouvrir ou se combiner dans l'analyse ; leur présentation compartimentée n'a d'autre but que de mieux identifier la ligne directrice de chacune. Elles s'avèrent toutes empreintes de psychologie, une psychologie intuitive et conjecturale dont l'ethnologue a peine à se passer face à ce type de situation.

Une première possibilité consisterait à arguer d'une différence de perception entre l'ethnologue étranger et les Birmans. Serait invoqué l'axiome culturaliste qui pose que les représentations collectives, agissant à la manière d'un prisme, commandent l'appréhension de la réalité ; que, par conséquent, les éléments qui, aux yeux d'un observateur extérieur, trahissent l'invraisemblance d'un phénomène, ne sont pas vus de la même façon, voire pas discernés, par les indigènes, dont les cadres mentaux engendrent une déformation perceptive. Cependant, dans le contexte du culte des quatre weikza, des Birmans distinguent la même chose que l'ethnologue, notamment la ressemblance entre deux des weikza et le médium ; ils regardent le culte comme une supercherie. En l'occurrence, les indigènes n'interprètent pas la réalité de manière identique, en vertu d'un schème collectif univoque (résultat prévisible, attendu que le mécanisme de l'interprétation fonctionne au niveau ouvert du croire - autorisant l'incrédulité - par-dessus le socle commun de la croyance). Écartée, par suite, la solution d'une surdétermination culturelle de l'expérience.

Une deuxième possibilité s'appuierait sur un postulat identique - le principe d'une illusion d'optique - pour le rapporter cette fois-ci à la conscience individuelle. Si la première hypothèse se plaçait au niveau de la croyance, la seconde se situe au niveau du croire. En gros, ceux qui deviennent les adeptes du culte se laisseraient machinalement abuser, en raison de leur volonté de croire et de la reconnaissance sociale dont jouit déjà le culte (effet boule de neige). Les choses 
se passent autrement néanmoins, puisque les adeptes s'avèrent sensibles à certaines invraisemblances des apparitions, invraisemblances qu'ils évoquent spontanément. Ainsi, ils constatent et mentionnent la ressemblance entre deux des weikza et le médium. Il serait infondé de leur prêter une totale cécité. Écartée, alors, la solution de l'aveuglement par le croire.

Une troisième possibilité, version amendée de la précédente, reposerait sur l'idée d'une rationalité propre au croire. Il y aurait bel et bien illusion, mais illusion sensée. Les croyants, plutôt que d'ignorer les invraisemblances, s'en serviraient avec une implacable logique pour mieux se conforter dans leur jugement. Les vices inhérents aux manifestations du surnaturel, loin d'entamer la crédibilité de ces manifestations, seraient un poids de plus jeté dans la balance de leur authenticité. Les adeptes du culte n'affirment-ils pas que la ressemblance entre deux des weikza et le médium est voulue, que c'est une façon parmi d'autres pour les weikza de fournir la preuve de leurs pouvoirs ? Pareillement, si un visiteur, lors d'une séance, a remarqué un bout de robe monastique de couleur ocre dépassant du costume blanc du weikza laïc, n'est-ce pas que le weikza, pour un motif connu de lui seul, a produit cette vision singulière afin d'empêcher le visiteur de croire ? Et ainsi de suite. En éliminant, à l'aide de raisonnements circulaires, les invraisemblances qui nourrissent le scepticisme des incrédules, les adeptes donneraient à la représentation plus qu'imparfaite des weikza une indiscutable consistance. Mais, quoique attentifs à des anomalies évidentes, et désireux d'en rendre compte, ils paraissent indifférents à nombre d'autres, qui sont pourtant flagrantes. Ces anomalies, ils ne se soucient pas de les relever, de les couvrir ou de les expliquer, alors même qu'ils insistent sur leur attitude de doute face aux weikza, sur leur examen minutieux des faits et sur leur exigence de preuves définitives. Ce contre quoi l'analyse bute, et que l'idée d'une rationalité propre au croire ne permet en rien d'éclairer, est le degré élevé de tolérance à l'invraisemblance et à l'incohérence en dépit d'un discours invoquant au contraire l'observation et la réflexion personnelles. Écartée, dans ce cas, la solution de la rationalité croyante, où toute chose trouverait une justification logique.

Une quatrième possibilité, enfin, engagerait à aborder les séances d'apparition comme un rituel, au sens de moment séparé des expériences de la vie quotidienne et conditionné par un mode cognitif particulier. Les individus, en ces occasions marquées, placeraient mécaniquement leur commutateur cognitif en position « rituel »; ils seraient dès lors en phase avec une représentation estimée en temps ordinaire dissonante, lacunaire ou fautive. De même qu'au théâtre, les acteurs et le public savent que ce qui se joue sur la scène est régi par des conventions spéciales mais n'en sont pas moins émus par l'action, les participants à un rituel n'attendraient pas que les faits se conforment à la règle ordinaire mais n'en seraient pas moins affectés. Tout deviendrait croyable, l'incroyable aussi bien que l'invraisemblable. La notion d'anomalie n'aurait aucune signification et ne serait d'aucune portée. Le rituel constituerait un monde à part, un segment 
de réalité cloisonné, une expérience décalée. Une telle hypothèse suppose que les participants soient d'accord d'emblée sur la nature de la situation. Peu importe qu'ils goûtent la circonstance ou non. L'essentiel est qu'il n'existe pas d'équivoque sur le cadre opératoire. Un rituel ne peut être autre chose qu'un rituel. Les participants, quand même ils l'auraient trouvé mal exécuté, ennuyeux ou décevant, quand même ils déploreraient son manque d'efficacité, ne diront pas que c'était un faux rituel, qu'on a voulu les tromper. Un rituel n'est jamais perçu comme artificiel. Or, pour ce qui concerne les apparitions des quatre weikza, la nature de la situation n'est pas définie a priori ; cette situation est en dehors de la sphère de l'action coutumière. L'objet de la représentation est précisément de persuader les spectateurs qu'ils ont affaire à une manifestation véridique du surnaturel et non à une imposture (ce qui ne réussit pas toujours). Qualifier les séances d'apparition de " rituel » reviendrait à occulter l'incertitude qui pèse sur le genre de la représentation et qui est au principe de son déroulement. Ce serait négliger la réaction que la situation suscite chez chaque individu, réaction capitale puisque croire signifie reconnaître la capacité des weikza à être un tremplin du salut. Ce serait éclipser qu'en l'occurrence le sens commun continue malgré tout de s'exercer, puisque les adeptes pointent du doigt quelques anomalies et s'attachent à les justifier. Écartée, pour toutes ces raisons, la solution de la modalité rituelle de l'expérience.

Finalement, aucune des quatre formules puisées dans l'escarcelle de l'anthropologie n'est satisfaisante. La question demeure toujours sans réponse : comment peut-on croire à une chose aussi peu crédible ? Si la clé du problème ne se trouve pas en aval, dans les eaux anthropologiques, elle se trouve sans doute en amont, aux sources indigènes. Qu'est-ce que les Birmans ont à nous apprendre sur leur relation au surnaturel, spécialement aux weikza?

\section{Une fiction bien réelle}

À chaque séance d'apparition, est présent un groupe d'une quinzaine de jeunes femmes du village qui procèdent à des récitations. Lorsque j'assistai pour la première fois à une séance, ces jeunes femmes firent montre de peu de respect à l'égard du premier weikza. Elles commentaient sa prestation à voix basse avec force plaisanteries et gloussements. L'une d'entre elles, les rappelant à l'ordre en me désignant, s'appliquait à mettre fin à ce discret chahut. Celui-ci reprenait bientôt de plus belle. Je devais vite m'habituer à leur indiscipline. Or ces villageoises qui se rient du weikza sont celles-là mêmes qui, pendant des années, consacrent nombre de leurs soirées à participer aux séances; qui, en outre, viennent en journée au monastère lorsque l'organisation d'une cérémonie ou le nettoyage des lieux l'exige. Ces jeunes femmes irrévérencieuses renvoyant le soidisant weikza à l'invraisemblance de son numéro sont les mêmes qui, interrogées sur le motif de leur substantielle implication dans la vie du culte, répondent avec 
conviction qu'elles y gagnent du mérite. De leur contribution, elles tirent aussi des bénéfices immédiats. Des adeptes leur font des cadeaux, en argent ou en nature. Le médium des weikza en emploie quelques-unes pour travailler aux champs de la famille et, à l'occasion, en emmène certaines avec lui en voyage ou en pèlerinage. Les deux ou trois plus âgées ont priorité, au moment de la fête annuelle du culte, pour installer des commerces de boissons et de nourriture. Ces bénéfices demeurent néanmoins restreints au regard de l'investissement demandé. Pour être aisément perceptible et si déconcertante, l'attitude à la fois sérieuse et railleuse de ces jeunes femmes n'en fait que mieux ressortir une certaine dualité du croire. Tout se passe comme si le croire combinait engagement absolu et lucidité aiguë, dévotion pleine et entière à l'être en lequel on croit et conscience de prendre part à l'élaboration d'une illusion collective.

Cela, au reste, coïncide avec une ambiguïté du discours commun. Les Birmans aiment à expliquer qu'en matière de weikza, "le croire est la base " (yonkyibmu akbyaykhan), c'est-à-dire que le croire fonde l'attribution à un personnage de la qualité de weikza. Ils font entendre, en d'autres termes, une théorie psychologique des cultes de weikza: un weikza est le produit du croire de ses adeptes. Les mêmes Birmans sont prompts, cependant, à donner les weikza particuliers en lesquels ils croient pour de "vrais weikza ", dont l'authenticité et les facultés sont attestées par des preuves objectives, indubitables. L'ambiguïté tient dans la coexistence de deux propositions a priori incompatibles: d'une part, c'est le croire qui fait le weikza; de l'autre, c'est le weikza qui fait le croire. Comment ces deux propositions, qui sont des interprétations, peuvent-elles s'articuler?

Un soir d'apparition, tandis que les weikza défilaient en procession parmi la foule enfiévrée, les fidèles se bousculant pour les atteindre, je vis une jeune fille, dont c'était la première visite, exprimer soudain la plus grande stupéfaction. Le weikza moine qui marchait en tête venait de passer près d'elle et elle s'était penchée vers lui pour recevoir sa bénédiction. Se redressant l'air ahuri, elle se plaça de façon à voir de près le visage du personnage - nul n'y prit garde, pas même le weikza. Celui-ci, après avoir avancé d'un pas ou deux, marqua un arrêt à cause de la foule qui faisait barrage. La jeune fille le rejoignit et scruta de nouveau son visage. Clairement, elle n'en croyait pas ses yeux. Qu'avait-elle aperçu ? La pièce de robe monastique qui recouvre et dissimule le crâne du weikza était mal disposée, et il était évident que le personnage, qui comme tout moine eût dû être rasé, jouissait d'une chevelure fournie (photo 12). L'expérience vécue par cette jeune fille, on peut, on doit (sauf à prendre les indigènes pour des idiots), supposer que tous les Birmans la font, à un degré ou un autre, quand ils découvrent le culte. Une chose est de croire aux superhommes, autre chose d'y être confronté, en chair et en os. L'écart entre ce que sont prétendument les weikza et la réalité brute ne saurait échapper à l'attention. La différence réside dans la réponse de chacun à la situation, une situation typique où la croyance aux weikza, une croyance au contenu assez vague et flou, s'expérimente au travers de 
sa manifestation empirique - des weikza de chair et d'os. En l'occurrence, deux réactions sont permises : soit crier à la supercherie et récuser le culte, soit reconnaître la mise en scène comme une composante de la manifestation du surnaturel. À mon sens, la seconde réaction est la condition de possibilité du croire. Aussi les adeptes du culte, alors même qu'ils voient une trappe s'ouvrir dans le faux plafond et un bras se tendre pour jeter une noix de coco ou des bougies, n'en présentent-ils pas moins les mains pour les recevoir. Ce qui se dit sans être énoncé, ce qui se négocie sans être débattu, parfois avec, parfois sans succès, au travers de la représentation des quatre weikza et de ses invraisemblances, pourrait être rendu par la formulation suivante: "Nous, les weikza, sommes une fiction, mais une fiction pleine de réalité et d'efficacité, parce que, par elle, s'accomplissent des choses essentielles pour vous, les adeptes, aussi bien que pour nous ». Les « choses essentielles » sont ce sur quoi l'ethnologue s'interroge normalement, ce qu'il s'évertue à discerner, la fin dernière de son travail d'analyse. Il cherche à mettre en évidence un ordre, des logiques, dont les indigènes, parce qu'ils vivent leurs idées et leurs pratiques, n'ont pas ou peu conscience. Pourtant, les indigènes semblent savoir ce qu'ils font, ou, plus exactement, ce qu'ils croient. Ils se connaissent mieux qu'on ne le pense.

Il n'est pas nécessaire de concevoir l'adhésion au culte comme le résultat d'un raisonnement articulé, d'un calcul - quelque chose comme : "Quoique tout cela soit une grossière supercherie, j'ai quand même intérêt à croire ». Au contraire, la définition de la situation et l'accord s'opèrent sourdement, et c'est ce qui donne à l'adhésion sa sincérité. Ou bien l'individu consent à pénétrer dans un ordre de réalité que spécifie une norme de vérité singulière : l'artifice, reconnu pour tel, fait partie intégrante du vrai. Ou bien il s'y refuse et verse dans l'incrédulité (sachant qu'en disqualifiant le culte, il ne s'interdit aucunement de croire en d'autres weikza). Le croire suppose en règle générale l'acceptation d'une part d'opacité. Lorsque, par exemple, un individu se met à être possédé, nul n'est en mesure de déterminer de manière indiscutable la vérité de la possession, même si nombre d'éléments sont brandis comme signifiants pour crédibiliser le phénomène ; même chose pour une apparition de la Vierge, etc. Le croire s'engendre à partir d'une carence de savoir qui est aussi non-dit. Un doute persiste inévitablement. Or le culte des quatre weikza a ceci de remarquable qu'il repose sur un dévoilement, dû notamment à ce fait inédit : l'apparition en chair et en os des weikza. Les discordances, tantôt infimes, tantôt majeures, en tout cas récurrentes, qui scandent le déroulement des séances d'apparition, sont fonctionnelles en ce qu'elles instituent un type distinctif de relation entre les weikza et les adeptes du culte. En laissant surgir des écarts renversants par rapport à l'expérience du surnaturel qu'ils s'efforcent de produire, en tombant presque dans un extraordinaire de pacotille comme s'ils voulaient affirmer haut et fort le caractère artificiel de la démonstration, weikza et adeptes font acte de distance pour définir ou rappeler les termes du contrat qui les lient - « nous croyons par convention, 
et ce croire est pleinement effectif ». Ils établissent ou renouvellent leur alliance en en soulignant et éprouvant les ressorts. Cela explique que l'invraisemblance soit supportée avec autant de sérénité.

Ainsi, l'invraisemblance de la représentation contribue à conférer à la séance d'apparition sa nature surnaturelle, elle est constitutive de sa spécificité, parce qu'elle désigne le croire comme l'assise première du culte, parce qu'elle souligne la dimension fictive, c'est-à-dire fabriquée, des weikza en tant que dimension essentielle à leur réalité. La démonstration n'est pas entièrement crédible et c'est pourquoi elle est croyable (si elle s'avérait lisse, c'est-à-dire n'appelait en rien une réaction, un mouvement conscient d'adhésion, elle appartiendrait à l'ordre de l'évidence partagée et non du croire). L'invraisemblance participe à l'anormalité de la situation : on sait qu'il y a mise en scène, savoir qui impartit aux faits leur consistance extraordinaire. L'invraisemblance joue, dans le phénomène du croire, un rôle structurant ; elle est une partie - mais une partie seulement - de ce qui rend possible l'expérience du surnaturel, car elle en travaille les conditions de réalisation et de perception. En un mot, elle fait signe. Cette signification ne recouvre pas la signification d'un autre type d'anomalies désignées par l'anthropologie cognitive comme caractéristiques de la religion et réunies sous le registre de la «contre-intuitivité » (expression qui qualifie, notamment, le fait que les entités surnaturelles sont toujours décrites comme dotées d'au moins une qualité remarquable qui les distingue, foncièrement, des humains auxquels elles s'apparentent par ailleurs) ${ }^{4}$. Les anomalies de la représentation, dans le cas présent, ne sont pas tant « contre-intuitives » que performatives : visibles, tangibles (au lieu de relever de simples énoncés), elles modulent l'expérience. Échappant au code de la contre-intuivité, ces anomalies ne signifient pas plus à la manière d'un texte de "commentaire métasocial ", selon une caractérisation célèbre de Clifford Geertz (1983 : 209). Elles ne sont pas, pour les acteurs, un moyen de glose sur leur monde, le monde du culte, le monde des quatre weikza. La fonction de l'invraisemblance est moins interprétative, au sens de Geertz, que productive : l'invraisemblance concourt au façonnage du monde du culte.

Une telle proposition, forte en ce qu'elle attribue beaucoup à l'invraisemblance, se démarque d'une lecture faible qui ne lui attribue rien. La seule alternative suggérée par certains parmi les premiers lecteurs de cette étude consiste à poser que les anomalies de la mise en scène n'affectent en aucune façon le processus du croire, qu'elles sont neutres : vues mais négligées et insignifiantes. Dans l'idéal, disent-ils, l'invraisemblance ne devrait pas être aussi flagrante ; malheureusement, il n'est pas d'expérience du surnaturel qui soit parfaite, la réalisation présente toujours quelques défaillances. Ainsi, les adeptes du culte croiraient malgré ces anomalies, et non avec elles. Étonnants croyants, capables, en dépit

4. Voir la postface de Jean-Pierre Albert à ce numéro. 
d'un souci exaspéré du vrai (un vrai qui met en jeu leur salut), de constater les ficelles grossières de la supercherie sans s'en émouvoir et sans en tenir compte ! C'est, de fait, la tendance des ethnologues à évacuer a priori le problème de l'invraisemblance qu'il convient à mon sens de remettre en cause. Si les indigènes sont sensibles au problème, de quel droit l'ethnologue le déclarerait-il indifférent ?

Arrêtons-nous un instant sur les orchestrateurs du culte, à savoir le médium et ceux qui coopèrent à la représentation (en aidant le weikza qui arrive en "volant » à grimper jusqu'à la fenêtre, en incarnant un weikza, en lançant les noix de coco et les bougies, etc.). Une chose surprend. À fréquenter ces individus, l'ethnologue se convainc qu'ils adhèrent à leur mise en scène, qu'ils croient aux weikza et à leurs pouvoirs. L'explication anthropologique courante - depuis Marcel Mauss et Henri Hubert au moins - veut que le magicien, sous l'effet de la croyance de la société dans son pouvoir, se mystifie lui-même et en arrive à simuler sans en avoir conscience; il serait, tout compte fait, aussi crédule que ses clients (Mauss, Hubert, 1973 : 88-89). Or il est impensable que les acteurs de notre culte oublient qu'ils jouent un rôle (pour reprendre la formule de Mauss et Hubert) tant celui-ci est activement fabriqué ; par ailleurs, les adeptes ne sont pas crédules, ils voient les tours de passe-passe nécessaires à la représentation. Mais si l'on fait du recours à l'artifice, plutôt qu'un pis-aller, une dimension inhérente à l'expérience du surnaturel, la difficulté disparaît. La conviction des orchestrateurs du culte devient un phénomène attendu, conforme à l'ordre des choses. On ne leur en demande d'ailleurs pas plus : il leur suffit, pour être probants, de jouer le jeu jusqu'au bout.

Mon hypothèse est en somme la suivante : croire implique de reconnaître le caractère de fiction du culte en faisant de ce caractère une partie nécessaire et intégrante de sa réalité. L'idée de fiction ne doit pas être confondue avec l'idée de faux; la fiction ressortit plutôt à la production d'une réalité singulière (le terme dérive du latin fictio, "action de fabriquer, de façonner, de créer » et « action de feindre, avec son résultat »). Il y a mille et une raisons de croire à une telle réalité, lesquelles ne m'intéressent pas ici. Mais, pour basculer dans cette attitude particulière, il faut d'abord que l'individu assimile l'invraisemblance de la représentation, c'est-à-dire renonce à l'expérience qu'il anticipait et en admette une forme différente, extraordinaire. Qui s'en tiendrait aux paramètres de l'expérience ordinaire demeurerait incrédule. Une expérience du surnaturel ne peut constituer une expérience normale et l'invraisemblance appartient à sa singularité.

Assurément, l'hypothèse est discutable. Les Birmans ne formulent presque jamais la chose dans des termes aussi univoques, en tout cas ceux qui croient. On ne s'en étonnera pas puisque l'invraisemblance concourt à la réalité du culte : ce qui est réel ne saurait être pris pour artificiel. Quoique spéculative et conjecturale, l'hypothèse ouvre une voie à l'interprétation, si nous voulons bien céder, à notre tour, aux sirènes de la circularité. Certains aspects du culte s'offrent sous un jour nouveau quand on les envisage à l'aune de cette solution. 
I42 - ARCHIVES DE SCIENCES SOCIALES DES RELIGIONS

\section{Les plaisirs du croire}

Les récits que font les Birmans à propos de leur adhésion au culte tournent autour des prodiges accomplis par les weikza. En rapportant comment ils ont été témoins de ces prodiges, comment ils les ont observés pour s'assurer de leur véridicité, les adeptes du culte - des hommes et des femmes de tous milieux sociaux, de toutes origines ethniques, de tous âges - justifient leur croire par une expérience personnelle. Aussi ces récits, énoncés à l'adresse de parents, de voisins, d'amis qui n'ont jamais assisté aux apparitions des quatre weikza et manifestent le plus souvent un scepticisme de principe à l'égard du phénomène, relèvent-ils, à première vue, d'une rhétorique de la preuve : un argument, articulé sous forme narrative (tableau de la ou des premières visites au monastère), certifie le phénomène. Les récits peuvent cependant être considérés différemment, comme des mythes individuels. Le héros est le narrateur, et le propos concerne la genèse, puis la poursuite de sa relation avec les weikza, une relation vitale puisque établie autour de la question du devenir de l'individu dans cette existence et les suivantes, et de son salut. La séance d'apparition reste le moment et le lieu clé de l'aventure, même s'il arrive que les weikza se révèlent préalablement au futur croyant, en rêve, chez lui, en route pour le lieu des apparitions en chair et en os, ou dans l'un des édifices du monastère peu avant la séance. Au cours de la séance, les weikza donnent l'occasion à un nouveau venu d'adhérer de manière à la fois formelle et personnelle au culte. En dehors des jours de fête, l'affluence au monastère est limitée. Les weikza ont tout loisir de s'adresser individuellement aux visiteurs, disciples de longue date ou inconnus. Ils en invitent certains dans la petite pièce située au coin avant droit de la salle, lieu à l'interface entre le monde humain et la Montagne des Dragonnes. Là, le néophyte se trouve en présence d'un ou de plusieurs weikza qui lui parlent, entendent ses vœux, l'assurent de leur protection, et lui remettent quelque objet (s'il devient adepte, il sera par la suite rappelé de temps à autre dans la pièce). Bref, les weikza s'efforcent de nouer avec le nouveau venu une relation personnalisée. Ils lui proposent de brancher son histoire individuelle sur une histoire collective, celle du culte, dont ils sont les maîtres d'œuvre. Soit ce branchement s'effectue et le récit subséquent de l'adepte accordera une place notable à l'expérience de l'extraordinaire et à l'entretien dans la petite pièce (ou à tout autre élément indiquant l'accointance avec les weikza) ; soit il échoue et le récit se fera incrédule, neutre et impersonnel. Quand un adepte se décrit éprouvant les weikza de diverses façons avant de s'autoriser à croire, il démontre au fond sa familiarité avec le culte en tant que fiction. Chacune des histoires qu'il relate dépeint sa confrontation avec le surnaturel et les réactions adéquates. Chacune parle de son aptitude à assumer la position de croyant. Chacune atteste la maîtrise qu'il a acquise du langage du culte, langage par lequel l'artifice est transformé en réalité. En énonçant son récit, en racontant par exemple comment il a vu un weikza voler au-dessus de sa voiture, il fait étalage de sa capacité fictionnelle, 
c'est-à-dire de sa faculté à produire de la réalité. Le croire se développe par apprentissage ; il se fabrique et s'exprime sur le mode d'une compétence.

S'observe au demeurant, chez une bonne partie des adeptes, une propension quasi frénétique à la mythification (ce que certains contempteurs du culte dénoncent comme une tendance à l'affabulation). À partir du moment où un individu a adhéré au culte, il devient l'un des participants et aussi l'un des auteurs de son histoire. Beaucoup s'en donnent à cœur joie, en multipliant les anecdotes les plus incroyables. La jouissance qu'il y a à mythifier touche à ce que j'appellerais l'ivresse de la représentation. La possibilité laissée à chacun de construire de la réalité à partir de la fiction, la possibilité de contribuer à la mise en forme de la réalité, notamment en élaborant un récit sur les weikza, paraît fort prisée. Et, en effet, qu'y a-t-il de plus satisfaisant pour un individu : avoir à subir ou reproduire passivement et anonymement la mise en œuvre d'une coutume, ou avoir la liberté de façonner une extraordinaire vérité ? Aussi bien, on pressent que la croyance elle-même évolue avec le croire : chaque individu, au travers de son expérience et des images qu'il s'en forme, s'équipe d'une représentation sur ce qu'est un weikza, représentation au contenu plus clair et précis qu'il n'était dans le cadre de la croyance initiale ; l'individu sait désormais ce qu'est un "vrai weikza». Le croire agit en retour sur la croyance, il lui donne une plus grande consistance mentale.

Au centre de cette construction en synergie qu'est le culte des quatre weikza, il y a le médium. Or, faire de l'invraisemblance une logique opératoire participant au fonctionnement et au succès du culte permet de mieux comprendre la fascination qu'exerce le personnage. Le médium des quatre weikza est un homme sans charisme de caractère; du moins est-il dénué de cette distinction et de cette autorité naturelles qui suscitent le respect. Il n'en impose pas. Il est toutefois seul capable de signifier avec autant d'assurance que le croire est une fiction, qui mérite d'être vécue comme réelle. C'est l'ambivalence du personnage, à la fois mystificateur et démystificateur, enchanteur et désenchanteur, qui est au fondement de sa grandeur. Qu'on songe à son usage du déguisement. Le travestissement auquel il recourt - pour être le weikza laïc puis l'un des weikza moines soutient la représentation; il s'avère pourtant trop sommaire pour faire oublier que cette représentation est fictive. Le costume autorise le médium, et à changer de rôle, et à conserver son identité. Le manque de vraisemblance de la représentation marque que le personnage connaît et affiche ce qui est d'ordinaire méconnu ou reconnu sur un mode plus équivoque au sein de la communauté, il prouve qu'il est maître du jeu. Et ce manque de vraisemblance fonctionne, pour cette raison, comme un élément moteur dans l'aura dont le médium bénéficie. La virtuosité du médium ne vient pas de ce qu'il assumerait certains traits emblématiques du rôle religieux (ascétisme, mysticisme) ; loin s'en faut. Elle vient de ce qu'il sait et donne à voir. Il n'y a, dès lors, rien de surprenant à ce que nul effort ne soit entrepris pour remédier aux accrocs de la mise en scène. Pourquoi, par 
exemple, chacun des weikza n'est-il pas incarné par un individu distinct, qui ne serait pas le médium ? Car ces accrocs semblent comme des signes volontaires à l'adresse du public.

L'attitude du personnage est en même temps la source d'une vive détestation par certains, qui perçoivent un danger dans son comportement. À souligner le caractère artificiel des quatre weikza, le médium ne remet-il pas en cause la croyance aux weikza en général ? Il n'est pas indifférent que certains moines l'accusent de "détruire la religion" (thathana hpyethsi-). S'ils entendent par là que ses activités sont contraires à la lettre et à l'esprit de l'enseignement bouddhique, l'accusation peut également être comprise comme une inquiétude quant aux implications de son attitude. En traitant si franchement l'objet de la croyance comme une fiction, le personnage sape les bases de la religion, il met à nu sa nature de construction humaine et sociale. Inquiétude, qui, tout bien considéré, semble inutile. Car si le médium sait que les quatre weikza sont une fiction, il sait aussi qu'ils sont une réalité. Il croit aux weikza.

Cette brève étude a exposé pourquoi et comment j'ai abouti à l'opinion que l'invraisemblance des manifestations du surnaturel était significative pour les indigènes et faisait question pour l'ethnologue; pourquoi et comment il s'est imposé à moi de considérer l'invraisemblance comme une dimension opératoire des expériences du surnaturel. Pour défendre une telle idée, il faut supposer, non sans témérité, que l'analyse d'un cas particulier, un cas hors du commun (même dans le contexte de la société concernée), est susceptible de revêtir une portée générale. Mais n'est-ce pas, justement, son caractère extrême qui fait l'intérêt de cet exemple par ce qu'il révèle d'un mécanisme du croire ailleurs moins sensible ? Quoi qu'il en soit, le principe qui a commandé aux développements du texte n'était pas de fournir une réponse jugée définitive et convaincante au problème envisagé. Il s'est agi, en courant de bon gré le risque de se tromper sur toute la ligne, de formuler et de soutenir jusqu'au bout, jusqu'à l'excès même, une hypothèse provocante afin de susciter la réflexion, de faire réagir. Je laisse à chacun le soin de juger de l'applicabilité d'une proposition finale qui se veut, pour mieux inviter à la critique, sans nuance.

Le croire, en tant que mode de relation au surnaturel, opère selon un régime de "fiction-réalité ». L'œuvre de fiction ne se définit pas par son contenu, par le fait qu'elle rapporte des choses vraies ou fausses. Elle se signale par le fait que s'établit un contrat entre son récepteur et son auteur, contrat par lequel le récepteur ne considère pas ce que l'auteur présente comme le reflet fidèle d'une réalité existante, passée ou présente ; une fiction est libre, non contrainte par les lois de la vérité et de la vraisemblance. Or, dans le cas du croire, les termes du contrat sont modifiés : les quatre weikza sont reconnus comme une fiction si effective qu'elle doit être, en même temps et plus encore, envisagée comme une réalité. Du moins pour ceux qui croient. Pour les autres, qui voient dans le culte une supercherie, la fiction reste fiction, et ceux qui y croient sont les victimes d'une 
illusion : ils ne distinguent pas entre fiction et réalité. Les incrédules, toutefois, s'ils n'adhèrent pas à cette fiction-ci, adhéreront à une autre. Ils deviendront les adeptes d'autres weikza.

Aussi conviendrait-il de s'appliquer à un renversement de perspective dans l'étude du croire. L'idée d'une opposition entre l'ethnologue et les indigènes, le premier regardant comme une fiction sociale signifiante et efficace ce que les seconds tiennent pour simple et pure réalité, doit laisser place à celle d'une appréhension commune, également paradoxale et ambiguë, des phénomènes surnaturels, qu'on résumerait volontiers en ces termes: quoique fictif, c'est parfaitement réel.

Guillaume ROZENBERG

Toulouse, LISST - Centre d'anthropologie sociale guillaume.rozenberg@gmail.com

\section{Bibliographie}

BAZIN Jean, 1991, "Les fantômes de Mme Du Deffand: exercices sur la croyance ", Critique. Revue générale des publications françaises et étrangères, XLVII/529-530, pp. 492-511.

Evans-Pritchard Edward E., 1937, Witchcraft, Oracles and Magic among the Azande, London, Oxford University Press.

FAVRET-SAADA Jeanne, 1995 [1977], Les mots, la mort, les sorts, Paris, Éditions Gallimard.

GeERTz Clifford C., 1983 [1972], "Jeu d'enfer. Notes sur le combat de coqs balinais », in Geertz C. C. (trad. par Louis Evrard), Bali. Interprétation d'une culture, Paris, Éditions Gallimard, pp. 165-215.

Hervieu-LÉger Danièle, 1993, La religion pour mémoire, Paris, Éditions du Cerf.

LENCLUD Gérard, 1990, "Vues de l'esprit, art de l'autre. L'ethnologie et les croyances en pays de savoir ", Terrain, "L'incroyable et ses preuves ", 14, pp. 5-19.

Mannoni Octave, 1969, "Je sais bien, mais quand même... ", in Mannoni O., Clefs pour l'imaginaire ou l'autre scène, Paris, Éditions du Seuil, pp. 9-33.

Mauss Marcel, Hubert Henri, 1973 [1950], «Esquisse d'une théorie générale de la magie » [1902-1903], in Mauss M., Sociologie et anthropologie, Paris, Presses Universitaires de France, pp. 1-141.

NeEdham Rodney, 1972, Belief, Language, and Experience, Oxford, Basil Blackwell.

Pouillon Jean, 1993, "Le cru et le su », in Pouillon J., Le cru et le su, Paris, Éditions du Seuil, pp. 17-36.

RozEnBERg Guillaume, 2005, Renoncement et puissance. La quête de la sainteté dans la Birmanie contemporaine, Genève, Éditions Olizane.

SouthWOLD Martin, 1979, «Religious Belief », Man, 14-4, pp. 628-644.

SPERBER Dan, 1982, "Les croyances apparemment irrationnelles ", in Sperber D., Le savoir des anthropologues. Trois essais, Paris, Hermann, pp. 49-85. 
I46 - ARCHIVES DE SCIENCES SOCIALES DES RELIGIONS

\section{Résumé}

L'article traite d'un culte bouddhique birman dédié à des personnages humains dotés de facultés surnaturelles, telle la faculté de voler. Ces personnages manifestent de diverses manières leurs facultés surnaturelles de façon à convaincre les fidèles qu'ils sont bien ce qu'ils prétendent être, à savoir d'authentiques weikza (catégorie birmane de superhommes). Or leur démonstration est marquée par des anomalies criantes qui contredisent la représentation qu'ils s'attachent à produire et soulignent son caractère de mystification. Le paradoxe est qu'en dépit de telles anomalies, un certain nombre de Birmans n'en croient pas moins que ces personnages sont de "vrais weikza". Faut-il alors ignorer, comme l'anthropologie l'a habituellement fait, le caractère invraisemblable de la représentation? Ou bien convient-il, au contraire, de prendre en compte l'invraisemblance et de chercher à l'expliquer, en l'intégrant comme dimension logique des manifestations du surnaturel ?

Mots-clés : Birmanie, cultes, weikza, surnaturel, croyance.

\section{Abstract}

This article deals with a Burmese Buddhist cult devoted to human figures endowed with supernatural faculties, such as the faculty of flying. These figures demonstrate in various ways their supernatural faculties in order to convince devotees that they are what they claim to be, that is, genuine weikza (a Burmese kind of supermen). However their demonstration implies obvious anomalies which contradict the representation they try to produce and underline its character of mystification. The paradox is that, despite such anomalies, numerous Burmese people nevertheless believe that these figures are "true weikza". Should we then ignore the unbelievable character of the demonstration, as anthropologists have so often done? Or should we, on the contrary, take this unbelievable character into account and look for an explanation, by considering this character as a logical dimension of supernatural manifestations?

Key words: Burma, cults, weikza, supernatural, belief.

\section{Resumen}

El artículo trata de un culto budista birmano dedicado a personajes humanos dotados de facultades sobrenaturales, por ejemplo la facultad de volar. Estos personajes manifiestan diversamente sus facultades sobrenaturales para convencer a los fieles que son lo que pretenden ser, a saber auténticos weikza (categoría birmana de superhombres). Sin embargo su demostración contiene anomalías importantes que contradicen la representación que intentan producir y subrayan su carácter de mistificación. La paradoja es que a pesar de tales anomalias, numerosos birmanos creen que estos personajes son "verdaderos weikza". ¿Podemos ignorar entonces, como lo hizo habitualmente la antropología, el carácter inverosímil de la representación ? ¿Conviene tomar en cuenta la inverosimilitud e intentar explicarla, integrándola como dimensión lógica de las manifestaciones del sobrenatural?

Palabras clave: Birmania, cultos, weikza, sobrenatural, creencia. 